\title{
Failure in Cervical Spinal Fusion and Current Management Modalities
}

\author{
Terence Verla, MD, MPH ${ }^{1}$ David S. Xu, MD ${ }^{1}$ Matthew J. Davis, BS ${ }^{2}$ \\ Edward M. Reece, MD, MBA, FACS, FAAP ${ }^{1,2}$ Michelle Kelly, PA- $C^{2}$ Mervin Nunez, PA-C ${ }^{2}$ \\ Sebastian J. Winocour, MD, MSc, FACS ${ }^{2}$ Alexander E. Ropper, MD ${ }^{1}$
}

${ }^{1}$ Department of Neurosurgery, Baylor College of Medicine,

Address for correspondence Alexander E. Ropper, MD, Department Houston, Texas

2 Division of Plastic Surgery, Michael E. DeBakey Department of of Neurosurgery, Baylor College of Medicine, 7200 Cambridge Street, Surgery, Baylor College of Medicine, Houston, Texas Suite 9A, Houston, TX 77030 (e-mail: alexander.ropper@bcm.edu).

Semin Plast Surg 2021;35:10-13.

\begin{abstract}
Keywords

- cervical pseudarthrosis

- spinoplastic reconstruction

- neurosurgery

- spine surgery

- plastic surgery
\end{abstract}

The cervical spine is a very mobile region of the spinal column, and its relationship to the head and the rest of the body makes it prone to injury. Common pathologies affecting the cervical spine include degenerative disc disease, spinal fractures from trauma, infection, and tumors. Indications for surgery depend on the underlying pathology, the presence of spinal instability, severe deformity, or neurologic compromise. These patients often present with cervical radiculopathy (nerve root impingement) or myelopathy (spinal cord compression) with features such as pain, numbness, tingling, weakness, and gait instability. Severe cases of instability and stenosis may lead to quadriplegia in some circumstances. Cervical spinal fusion can be indicated to treat these pathologies.

\section{Indications for Anterior versus Posterior Approach}

The decision to approach the spine anteriorly or posteriorly depends on multiple factors, including the location of the pathology, the involved spinal levels, the area of the bony spinal column ring involved, and access to the diseased area. Cervical spinal fusion has been performed for decades as treatment for patients with symptomatic pathologies. Anterior cervical discectomy and fusion (ACDF) is one of the most common spine fusion procedures. ${ }^{1}$ Access for ACDF is achieved by approaching the cervical spine from the anterior neck, dissecting a plane medial to the sternocleidomastoid muscle and carotid sheath, and retracting the central strap (c) 2021. Thieme. All rights reserved. Thieme Medical Publishers, Inc., 333 Seventh Avenue, 18th Floor, New York, NY 10001, USA
DOI https://doi.org/ $10.1055 / \mathrm{s}-0041-1722853$. ISSN 1535-2188. Reece, MD, MBA, FACS, FAAP
Reconstruction; Guest Editor: Edward M. 
muscles, trachea, and hypopharynx/esophagus. In common cases of degenerative discs resulting in symptomatic nerve root of spinal cord compression, the intervertebral disc material is then removed, and an allograft bone or artificial spacer filled with bone graft material is inserted into the empty disc space to promote fusion between the adjacent cervical vertebrae. To maximize the probability of fusion, the fused segments are immobilized by plates and screws. However, in some cases where the anterior spine cannot be accessed as such, or nature of pathology dictates a posterior approach, dissection is performed down to the posterior spinal elements (spinous process, lamina, lateral mass, etc.) where instrumentation is placed laterally for fixation. The central canal is then decompressed with laminectomies. The facets and surrounding bony elements are then decorticated, and bone-graft material is placed on exposed surfaces to promote fusion.

Prior to the widespread adoption of internal fixation, spinal fusion was performed for patients with moderate spinal deformity with decortication of the fusion bed and application of autograft. The patient's neck would then be immobilized in a halo device or other external orthosis until arthrodesis was achieved. The principles and goals of fusion are no different in the current day, except the material advances in instrumentation have allowed not only internal fixation but also the ability to correct more significant spinal deformities.

\section{Failure in Cervical Spine Fusion}

Despite the efforts to promote osseous fusion, pseudarthrosis (failed fusion) remains a significant complication that should be anticipated in select cases and managed accordingly. The rates of failed fusion in the cervical spine are quite variable, ranging from 2 to $30 \%$, with this variability partly due to the inconsistent diagnostic measures used postoperatively and also the asymptomatic nature of many patients with pseudarthrosis. ${ }^{2-4}$ However, in symptomatic patients (with pain being the main complaint), revision surgery is often required, as reoperation has a tremendous effect on patient quality of life outcomes; but revision surgery also comes with a significant economic burden on the healthcare system. ${ }^{2}$ As such, the initial fusion operation should be maximally optimized.

\section{Risk Factors for Failure of Fusion in Cervical Spine}

There are several factors that affect the degree of fusion following cervical spine surgery. The exact etiology of pseudarthrosis remains unclear and is certainly multifactorial. Factors influencing fusion can be divided into two broad categories: patient-related factors and surgical factors. Common patient-related risk factors include low bone mineral density (e.g., osteoporosis), chronic steroid use, smoking, diabetes, poor nutrition, and obesity. ${ }^{5-8}$ Other metabolic disorders-especially those that affect osteoblast and osteoclast homeostasis and bone formation-can precipitate failure of fusion. Some of these factors are modifiable and are often optimized preoperatively to enhance outcomes. Surgical factors vary and include surgical approach (anterior vs. posterior vs. combined anterior-posterior fusion), multilevel fusion, lack of thorough disc space preparation, the use of anterior plating, and the type of bone graft material utilized (allograft vs. autograft). ${ }^{9-11}$ Obtaining a durable fusion at the craniocervical junction is especially challenging given the majority of normal head and neck motion is a result of the mobile occipital-C1 and C1-2 joints, as well as the paucity of bony posterior elements to decorticate as a fusion bed. These risk factors, alone or in combination, are just a subset of the multidimensional phenomenon underlying failed cervical spinal fusion.

\section{Diagnosis of Cervical Spine Pseudarthrosis}

Some cases of pseudarthrosis are found incidentally on routine postoperative imaging especially when the patient is asymptomatic. For symptomatic patients presenting with new neck pain, radiculopathy or myelopathy, the degree of osseous fusion in the cervical spine can be assessed with computed tomography (CT) scan or plain X-rays. On a thin-cut CT image, fusion is indicated by the presence of bridging bone formation between vertebral bodies, facet joints, or in the posteriorlateral elements of the cervical spine. On X-rays, bridging bone can sometimes be seen in the vertebral body-graft interface, but the reliability of this finding as a measure of fusion is not highly reproducible. Moreover, it is rather challenging to decipher fusion of posterior elements on plain X-rays given the small size of the posterior facet joints. For severe cases of failed fusion, the presence of loose or displaced fixation hardware or dynamic motion on flexion-extension X-rays could be useful in diagnosis. ${ }^{4}$

\section{Management of Fusion Failure}

For asymptomatic patients with incidental pseudarthrosis alone, conservative management is often the preferred treatment option. Despite a failed fusion, these patients may have a stable nonunion with fibrous tissue bridging the bone. In many cases, this fibrous tissue will prevent abnormal translation of adjacent segments and prevent further canal compromise or hardware loosening. Optimization of patient-related risk factors such as smoking, poor nutrition, osteopenia/osteoporosis, obesity, diabetes, and others may also improve outcomes. By addressing these comorbidities, patients also become better suited for enhanced outcomes in the event of a revision surgery.

Surgery for pseudarthrosis is usually indicated for symptomatic patients. Based on the initial surgical approach (anterior or posterior), the decision is carefully made on how to address the failed fusion. There is a benefit to re-exploring the anterior approach during the revision surgery because it offers the ability to explore the pseudarthrosis site, to change the bone-graft material, to utilize autograft, and to implant a more stable biomechanical construct to enhance fusion. Alternatively, a posterior fixation and fusion following a failed anterior approach offers the advantage of a native bony 


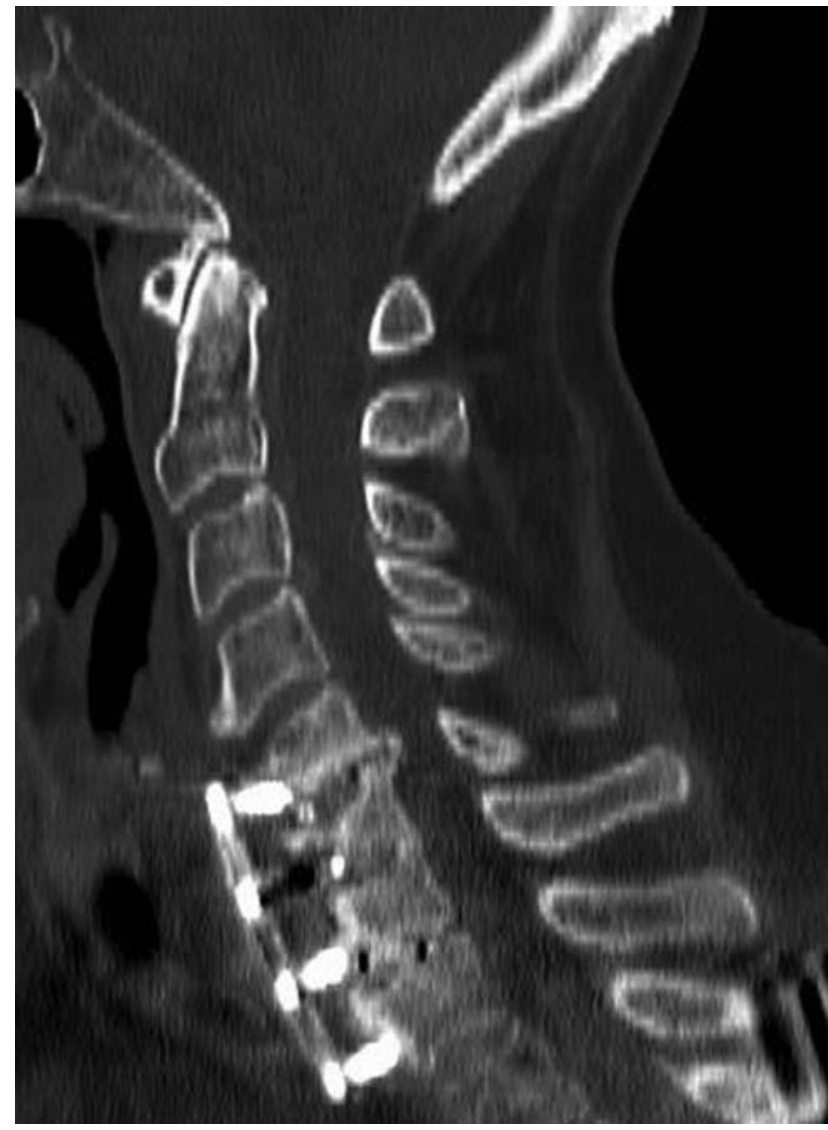

Fig. 1 Computed tomography of the cervical spine showing anterior cervical discectomy and fusion with plating at C5 to T1, pseudarthrosis (failed fusion), subsidence (interbody cage migration), and loosening of hardware (screws and plate). interface for fusion, and the literature suggests evidence of improved fusion rates and patient outcomes when this approach is taken. ${ }^{5,12-14}$ In some advanced cases of failed fusion with segmental instability and associated kyphotic deformity, a combined anterior-posterior approach may be suitable to achieve a durable arthrodesis.

\section{Intraoperative Adjuncts to Enhance Spinal Fusion}

The choice of intraoperative adjuncts to enhance cervical spine fusion depends on the surgical approach. For anterior cervical fusion, studies have shown that the presence of autograft as the choice of bone graft yields higher fusion rates compared with allografts. Allografts such as cadaveric fibular struts are frequently used in cervical corpectomy surgeries to play a role in structural support and fusion augmentation, and this approach is known to promote fusion more than expandable cages. In rare cases, a fibular free flap has historically been used for large defects at high risk of pseudarthrosis.

For posterior cervical spinal fusion cases, fusion is typically enhanced by adequate decortication of the facet joints and posterior bony elements, a combination of crushed autografts and artificial bone graft extenders or allograft, and the use of structural allografts. Autologous iliac crest bone graft is an excellent option for both anterior and posterior cervical fusion graft material. However, there are morbidities associated with the harvest site, including infection, pain, and the need for an additional surgical site.

Both autologous and allogenic bone grafts lack the vascularity that is critical to postoperative bone formation and subsequent fusion. Despite iliac crest grafts being osteoconductive, osteoinductive, and osteogenic, the absence of the

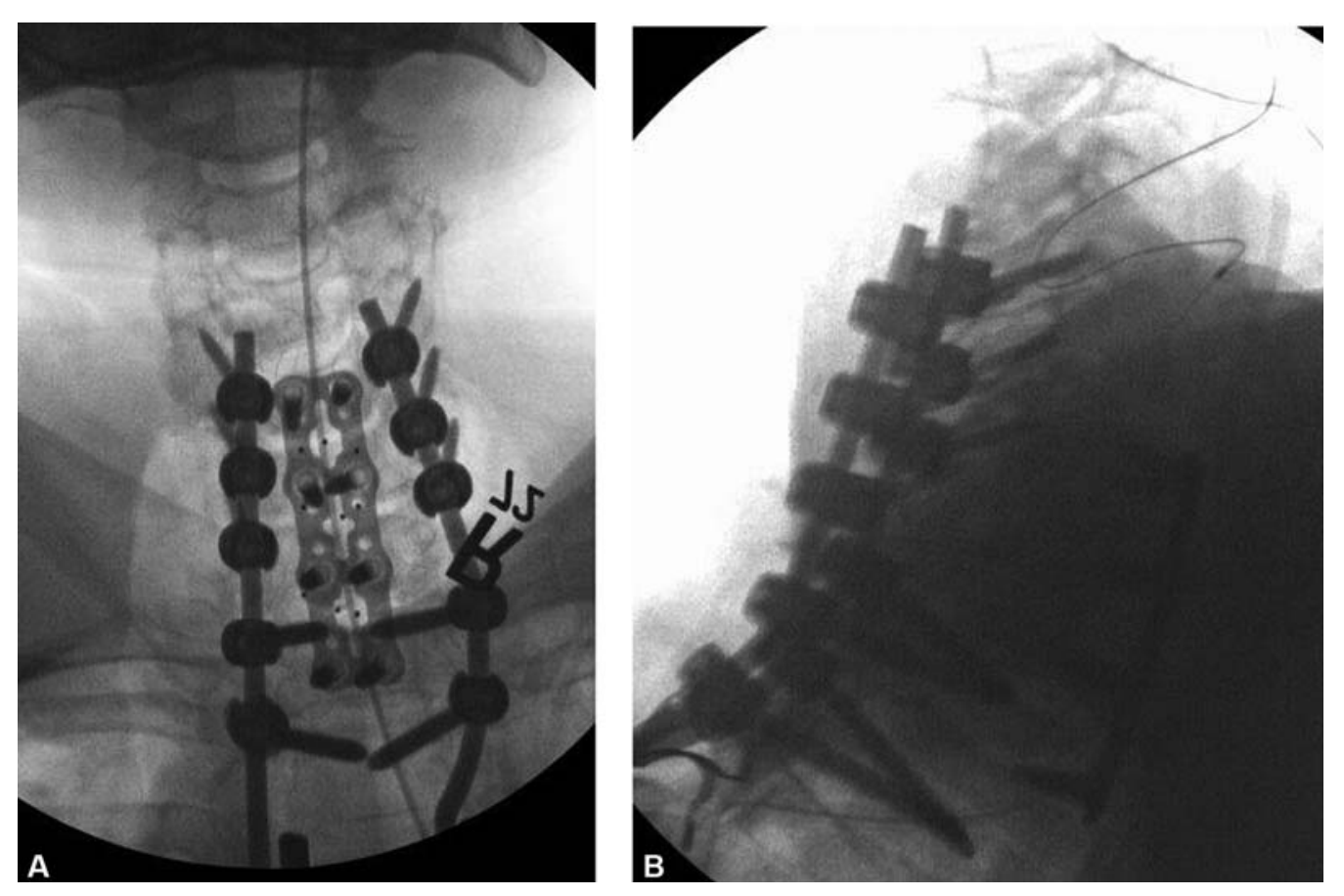

Fig. 2 Patient underwent C4 to T2 decompression, fixation, and fusion with the use of autografts and allografts for fusion. (A) Anterior/posterior $\mathrm{X}$-rays and (B) lateral X-rays showing instrumentation from C4 to T2. 
graft's vascularity after harvest is a limitation when utilized in the cervical spine. A novel, vascularized approach to augment arthrodesis in the cervical spine is the utilization of vascularized bone grafts (VBGs). Common sites of harvest for cervical spine fusion include the occipital VBG for high cervical and occipitocervical fusion, the medial scapula VBG for midcervical fusio, and rib VBGs for lower cervical or cervicothoracic fusion. Advantages of VBGs include their cellular viability, structural integrity, revascularization and remodeling of adjacent bone, greater resistance to infection and higher, and faster fusion rates. Access to these VBGs is made mostly through the same surgical incision without any significant added morbidity.

\section{Case Illustration}

A 70-year-old female with a history of rheumatoid arthritis, chronic back pain, and scoliosis presented with cervical radiculopathy. She underwent a multilevel ACDF at C5-T1 to treat the cervical stenosis causing compressive radiculopathy and myelopathy. Poly-ether ether ketone interbody spacers filled with bone graft were placed in the disc spaces to facilitate intervertebral fusion. She had an excellent initial neurological recovery after this surgery. However, 3 years later she presented with neck pain and recurrent cervical radiculopathy. CT scan of the cervical spine showed pseudarthrosis in the anterior cervical spine with loosening of the screws and plating and also subsidence (migration) of the cage into the vertebral bodies ( - Fig. 1). Given her symptomatic pseudarthrosis, together with cervical-thoracic deformity, a revision surgery was performed. An anterior approach was avoided given the number of vertebral bodies involved, compromised bony elements, and the risk of injury to surrounding structures from exposure (vessels, esophagus, laryngeal nerves, etc.). She therefore underwent a posterior approach involving decompression and instrumented fusion from C4 to T2 (-Fig. 2) with utilization of biologics (allograft cellular bone graft), allografts, and locally harvested autograft. Surgery was without complications and she continues to recover.

The complication of pseudarthrosis following this patient's cervical fusion suggests that perhaps a more robust operation should have been initially chosen to optimize her potential for successful fusion. VBGs could be utilized in settings such as these to augment cervical fusion.

\section{Conflict of Interest}

Dr. Ropper receives consulting fees from Globus Medical and Stryker, but they have no conflict with this report.

\section{References}

1 Saifi C, Fein AW, Cazzulino A, et al. Trends in resource utilization and rate of cervical disc arthroplasty and anterior cervical discectomy and fusion throughout the United States from 2006 to 2013. Spine J 2018;18(06):1022-1029

2 Pennington Z, Mehta VA, Lubelski D, et al. Quality of life and cost implications of pseudarthrosis after anterior cervical discectomy and fusion and its subsequent revision surgery. World Neurosurg 2020;133:e592-e599

3 Lowery GL, Swank ML, McDonough RF. Surgical revision for failed anterior cervical fusions. Articular pillar plating or anterior revision? Spine 1995;20(22):2436-2441

4 Cannada LK, Scherping SC, Yoo JU, Jones PK, Emery SE. Pseudoarthrosis of the cervical spine: a comparison of radiographic diagnostic measures. Spine 2003;28(01):46-51

5 Phillips FM, Carlson G, Emery SE, Bohlman HH. Anterior cervical pseudarthrosis. Natural history and treatment. Spine 1997;22 (14):1585-1589

6 Bolesta MJ, Rechtine GR II, Chrin AM. One- and two-level anterior cervical discectomy and fusion: the effect of plate fixation. Spine J 2002;2(03):197-203

7 Hilibrand AS, Fye MA, Emery SE, Palumbo MA, Bohlman HH. Impact of smoking on the outcome of anterior cervical arthrodesis with interbody or strut-grafting. J Bone Joint Surg Am 2001;83(05):668-673

8 Lau D, Chou D, Ziewacz JE, Mummaneni PV. The effects of smoking on perioperative outcomes and pseudarthrosis following anterior cervical corpectomy: clinical article. J Neurosurg Spine 2014;21(04):547-558

9 Samartzis D, Shen FH, Matthews DK, Yoon ST, Goldberg EJ, An HS. Comparison of allograft to autograft in multilevel anterior cervical discectomy and fusion with rigid plate fixation. Spine J 2003;3(06): 451-459

10 Wang JC, McDonough PW, Endow KK, Delamarter RB. Increased fusion rates with cervical plating for two-level anterior cervical discectomy and fusion. Spine 2000;25(01):41-45

11 Wang JC, McDonough PW, Kanim LE, Endow KK, Delamarter RB. Increased fusion rates with cervical plating for three-level anterior cervical discectomy and fusion. Spine 2001;26(06):643-646, discussion 646-647

12 Brodsky AE, Khalil MA, Sassard WR, Newman BP. Repair of symptomatic pseudoarthrosis of anterior cervical fusion. Posterior versus anterior repair. Spine 1992;17(10):1137-1143

13 Farey ID, McAfee PC, Davis RF, Long DM. Pseudarthrosis of the cervical spine after anterior arthrodesis. Treatment by posterior nerve-root decompression, stabilization, and arthrodesis. J Bone Joint Surg Am 1990;72(08):1171-1177

14 Kuhns CA, Geck MJ, Wang JC, Delamarter RB. An outcomes analysis of the treatment of cervical pseudarthrosis with posterior fusion. Spine 2005;30(21):2424-2429 